\title{
The Correlation between Dysphagia with Serum Albumin Level and Activities of Daily Living Improvement among Stroke Rehabilitation Patients: A Retrospective Study
}

\section{Kazuya Ikenishi*}

Chiba Institute Science Choshi, Chiba prefecture, Japan

*Corresponding author: Kazuya Ikenishi, Chiba Institute Science Choshi, Chiba prefecture, Japan, Tel: +81 0479-30- 4588; E-mail: kikenishi@cis.ac.jp

Received date: Feb 12, 2019; Accepted date: Mar 07, 2019; Published date: Mar 14, 2019

Copyright: (C) 2019 Ikenishi K. This is an open-access article distributed under the terms of the Creative Commons Attribution License, which permits unrestricted use, distribution, and reproduction in any medium, provided the original author and source are credited.

\begin{abstract}
Background: Recent years have witnessed an upsurge in the interest in the correlation between nutritional condition and rehabilitation of stroke patients. Dysphagia is one of the neurological manifestations, which markedly affects the nutrient intake of stroke patients. However, previous studies did not consider dysphagia as a variable. If the presence of dysphagia correlated with the serum albumin level and activities of daily living (ADL) of stroke patients, these studies could have contributed to enhancing nursing care for rehabilitation stroke patients. This study aims to investigate the correlation between dysphagia with the serum albumin level and the functional independence measure (FIM) of stroke rehabilitation patients.
\end{abstract}

Methods: In this retrospective study, we reviewed medical records in a rehabilitation hospital located in the Kanto area of Japan, from November 1, 2018 to January 31, 2019. From medical records, we collected data about age and sex, type of stroke, location of the stroke, serum albumin level, and the presence or absence of dysphagia. Using the univariate analysis, we evaluated the correlation between dysphagia and two variables-serum albumin level and FIM score.

Results: The study cohort comprised 22 stroke patients [presence of dysphagia (dysphagia group), 16; and absence of dysphagia (no dysphagia group), 6]. Regarding the serum albumin level, no significant was noted between groups. However, the dysphagia group's FIM score and variation were markedly higher than the no dysphagia group.

Conclusions: Regarding stroke patients in a recovery phase rehabilitation ward, comparison of patients with dysphagia and no dysphagia reveals the following: FIM scores are markedly higher; serum albumin levels are not markedly different groups; and dysphagia stroke patients' average serum albumin level declines after 1 month.

Keywords: Stroke; Dysphagia; ADL; Rehabilitaiton nursing; Serum albumin

\section{Introduction}

Dysphagia is one of the neurological manifestations, which markedly affects the nutrient intake of stroke patients. Regrettably, dysphagia is highly prevalent in stroke patients. Recent years have witnessed an upsurge in the interest in the correlation between nutritional condition and rehabilitation of stroke patients $[1,2]$. To date, several studies have reported that the improvement of the condition correlated with the enhancement of the stroke rehabilitation effect $[3,4]$. Better nutrition condition of stroke patients with dysphagia can be obtained through common medical practices like tubal feeding and gastric fistula, except for facilitating oral ingestion. However, when evaluating using the serum albumin level, enhancement of the nutrition condition of stroke patients did not seem to be achieved easily because it reported a declined level after admission to a rehabilitation hospital and recovered/elevated until discharge [5]. For the declined levels, this researcher considered that acute stroke patients were obliged to visit a hospital immediately. Moreover, with the declining serum albumin level of a stroke patient, protein hyper metabolism might worsen because of implementing rehabilitation.
Furthermore, some studies have reported the correlation between malnutrition and poor outcome of rehabilitation $[6,7]$.

In this study, we analyse the serum albumin level of stroke patients. Serum albumin is characterized by a half-life of 20 days, compared with transthyretin and retinol-binding protein, and is affected by liver/ renal disease and inflammation. Thus, serum albumin is not a suitable indicator for evaluating acute patients' nutrition condition change in the short term [8]. Conversely, if the condition of convalescent patients is calm, the nutrition condition could be assessed using the albumin level.

The findings mentioned above were critical for not only physical therapists and occupational therapists but also nurses who provided care and rehabilitation for patients. For example, if patients display a lower level of serum albumin with protein hyper metabolism, the implementation of walking rehabilitation with nursing aid might diminish their muscle mass further. Reportedly, the nutrition level of stroke patients dropped briefly in the acute phase [9]. In addition, the serum albumin level of acute stroke patients was markedly different between general diet and dysphagia diet, as well as tubal feeding at the hospital for 7 days, with the general diet group exhibiting the highest level [9]. Furthermore, the lower level at the acute hospital was for 7 
days than one at admission among all groups; however, this study did not analyse the correlation between dysphagia and activities of daily living (ADL) [9].

A study established a significant correlation between the serum albumin level and ADL [10]. In addition, a study investigated the correlation between the serum albumin level of patients with acute cerebral infarction at admission and the functional outcome (modified Rankin Scale, mRS) at hospital for 7 days, clarifying that the higher the albumin level, the lower score of the mRS; thus, the study revealed that with the higher albumin level, the functional outcome of patients with acute cerebral infarction was better compared with patients with lower levels [11]. Another study revealed a markedly positive correlation between the serum albumin level at admission and ADL (Barthel Index, BI) at discharge among stroke patients in a rehabilitation hospital after discharge from an acute hospital [10]. However, these studies did not include dysphagia as a variable. If the presence of dysphagia correlated with the serum albumin level and ADL of stroke patients, these studies could have contributed to enhancing nursing care for rehabilitation stroke patients.

This study focuses on the variation in the serum albumin level and ADL of stroke patients with dysphagia. Hence, regarding the albumin level and ADL, this study aims to compare stroke patients with/ without dysphagia. In addition, this study aims to investigate the correlation between dysphagia with the serum albumin level and the functional independence measure (FIM) of stroke rehabilitation patients.

\section{Methods}

In this retrospective study, conducted between November 1, 2018 and January 31, 2019, we reviewed medical records of a rehabilitation hospital after obtaining consent from individual subjects or their families. This research protocol was approved by the Chiba Institute of Science (No.30-1) and a rehabilitation hospital.

The inclusion criteria for patients were as follows: aged 20 years; and diagnosed with cerebral infarction or cerebral haemorrhage, subarachnoid haemorrhage. From medical records, we collected data about age and sex, type of stroke (cerebral infarction, cerebral haemorrhage, subarachnoid haemorrhage), location of stroke (right, left of cerebrum, or other), serum albumin level (admission, one month later; variation=level at one month later-level at admission), the presence or absence of dysphagia, and FIM score (admission, one month later; variation=score at one month later-score at admission). As collecting these data, we checked any omissions or errors on several record items of interest only.

We used the FIM scale to assess ADL of stroke patients at admission and after 1 month in the rehabilitation hospital. The FIM scale comprises 13 physical items (motor subscale) and five cognitive items (cognitive subscale). Each item was scored from one (total dependence) to seven (complete independence) based on the assessment of rehabilitation therapists in the study hospital. The total score of FIM was 18-126 points; the higher score implied higher independence of patients. Furthermore, we used the total point of FIM in analyses.

We examined the serum albumin level for assessing the nutrition condition of inpatients. The albumin level and the FIM score were collected at admission and one month after hospitalization. In addition, we analysed the variation of these variables, which was evaluated as follows:

Variation of serum albumin level = one month later albumin leveladmission albumin level

Variation of FIM score $=$ one month later FIM score-admission FIM score

There were some reasons for measuring the two data at 1 month later after admission. The features of serum albumin level have about half-life of 20 days and doesn't big change in short term [8]. The level at admission in the rehabilitation hospital was showed at acute term that patients received medical treatment in an acute hospital. However, the serum level at 1 month later showed nutrition condition receiving some cares and treatments in the rehabilitation hospital. Moreover, an improvement of the condition was important for good rehabilitation outcome. Therefore, we investigated the serum level and variation at I month later. Another reason about 1 month later was a period of routine blood examination in the hospital. Because the examination was seemed to do once every month at most rehabilitation hospitals, it was considered that we could obtain useful result by analysis on these data for using in at other rehabilitation hospitals. FIM score at 1 month later was used by same reasons.

Moreover, the presence of dysphagia was assessed by doctors and therapists from patients' medical records.

The study rehabilitation hospital was located in the Kanto area of Japan and had three recovery phase rehabilitation wards that adopted an approach of a multidisciplinary team comprising a doctor, nurse, physical therapist, occupational therapist, speech therapist, and nutritionist. These wards had approximately 160 beds, 170 nurse and care staff, and 110 therapists. Furthermore, the hospital accepted 600 patients in 2017 . Nearly $90 \%$ were discharged annually; this rate was higher than $78 \%$ of the national average in 2017.

In addition, the hospital provided dysphagia stroke patients with care to promote oral ingestion such as help with eating and preparation of meal environment, swallowing exercise by nurses, therapists, and care staff. Furthermore, patients with difficulty in oral ingestion and inadequate nutrient intake by only ingestion were offered some nutrition through tubal feeding or gastric fistula.

A speech therapist provided therapy to inpatients with rehabilitation regarding swallowing and/or voice disorder. A nutritionist and doctor adjusted diet for inpatients, including meal form, dietary composition, and calorie intake. The multidisciplinary team endeavoured to enhance the nutrition condition of patients. Moreover, the team held a meeting for explaining about rehabilitation and life condition in the hospital, confirming the feature policy that patients and families considered, hearing our their concerns, and return to their home.

Using the univariate analysis, we elucidated the correlation between dysphagia and two variables-serum albumin level and FIM score. We analysed age, serum albumin level, FIM score, and variation in the FIM score using the Student's t-test. In addition, sex, stroke type, and variation in the serum albumin level were assessed using the Fisher's exact test. The variation in the albumin level was categorized into decrease or non-decrease 1 month after admission for this analysis. As all enrolled subjects displayed elevated FIM score after 1 month of admission, the Fisher's exact test was not used to analyse the FIM variation. We set the statistical significance at $\mathrm{P}<0.05$. 


\section{Results}

In this study, all subjects were 22 stroke patients (11 male and 11 female; age: $44-95$ years; mean age: 75.27 years). The stroke type was 14 cerebral infarction, eight cerebral haemorrhage, and zero subarachnoid haemorrhage. The locations of stroke were eight right, 14 left of the cerebrum, and zero other. While 16 patients were with dysphagia (dysphagia group), 6 were without it (no dysphagia group; Table 1). No subject had liver and renal disease in his/her medical history, and CRP was below $0.30 \mathrm{mg} / \mathrm{dL}$ at admission.

\begin{tabular}{|c|c|c|c|c|c|}
\hline Variable & Unit & No Dysphagia $(n=6)$ & Dysphagia $(n=16)$ & Test statistic & p-value \\
\hline Age $^{*} \operatorname{man} \pm S D$ & Years & $71.83 \pm 14.78$ & $76.56 \pm 12.90$ & $t=-0.737$ & 0.47 \\
\hline $\operatorname{Sex}^{* *}$ (Men/Women) & Person & 43470 & 43744 & N/A & 0.149 \\
\hline Style of stroke ${ }^{* *}(\mathrm{Cl} / \mathrm{CH})$ & Person & 43500 & 43626 & N/A & 1 \\
\hline Location $^{\star \star}$ (Right / Left) & Person & $0 / 6$ & 43685 & N/A & 0.051 \\
\hline Serum albumin level at admission ${ }^{*}$ mean \pm SD & $\mathrm{g} / \mathrm{dl}$ & $3.867 \pm 0.43$ & $3.638 \pm 0.49$ & $t=1.001$ & 0.329 \\
\hline Serum albumin level at 1 month later ${ }^{*}$ mean $\pm S D$ & $\mathrm{~g} / \mathrm{dl}$ & $3.95 \pm 0.46$ & $3.59 \pm 0.43$ & $t=1.730$ & 0.099 \\
\hline Variation of serum albumin level ${ }^{*}$ mean $\pm S D$ & $\mathrm{~g} / \mathrm{dl}$ & $0.08 \pm 0.29$ & $-0.05 \pm 0.37$ & N/A & N/A \\
\hline No decline / Decline ${ }^{* *}$ & Person & & & N/A & 0.635 \\
\hline FIM score at admission ${ }^{*}$ mean \pm SD & Points & $57.33 \pm 4.55$ & $41.81 \pm 14.52$ & $t=2.538$ & 0.02 \\
\hline FIM score at 1 month later ${ }^{*}$ mean $\pm S D$ & Points & $91.00 \pm 17.30$ & $58.56 \pm 20.82$ & $t=3.388$ & 0.003 \\
\hline Variation of FIM score* mean \pm SD & Points & $33.67 \pm 16.85$ & $16.75 \pm 12.59$ & $t=2.565$ & 0.019 \\
\hline
\end{tabular}

Table1: Results.

The average age of the dysphagia group was higher than the no dysphagia group, but not statistically significant $(\mathrm{P}=0.470)$. In addition, sex $(\mathrm{P}=0.155)$ and stroke type $(\mathrm{P}=1.000)$ were not significant between groups. Regarding the location, all patients in the no dysphagia group were left, while those in the dysphagia group were divided by half, with no significant differences between groups $(\mathrm{P}=0.051)$.

Regarding the serum albumin level, no significant was noted at both admission $(\mathrm{P}=0.329)$ and one month later $(\mathrm{P}=0.099)$. The serum albumin level after one month declined in two patients in the no dysphagia group and seven in the dysphagia group. The Fisher's exact test revealed no significant differences between groups in the negative variation of serum albumin level $(\mathrm{P}=0.635)$.

\section{Discussion}

This study investigated the correlation between dysphagia and serum albumin level/FIM of stroke rehabilitation patients. The presence of dysphagia was not associated with the albumin level markedly.

Comparing the mean albumin level at admission with that 1 month later, the albumin level of the no dysphagia group increased from 3.867 to $3.950 \mathrm{~g} / \mathrm{dL}$. Conversely, the level of the dysphagia group decreased from 3.638 to $3.590 \mathrm{~g} / \mathrm{dL}$. Despite that, it was not significant between groups because of the wide confidence interval due to the small sample size.

Regarding the declined albumin level, the Fisher's exact test revealed no significant difference between groups. However, the number of subjects with the declined level was 7 of $13(43.8 \%)$ in the dysphagia group, and 2 of $6(33.3 \%)$ in the no dysphagia group. In addition, as the average level of the no dysphagia group increased by $0.08 \mathrm{~g} / \mathrm{dL}$ but the dysphagia group decreased by $0.05 \mathrm{~g} / \mathrm{dL}$, the between-group difference tended to expand. Thus, further investigation is warranted to explore the reason.

Our literature search failed to yield any study that reported the serum albumin level of stroke patients declining 1 month after admission in recovery phase rehabilitation wards. Some studies revealed that the nutrition condition declined in the acute period or from the acute phase to the rehabilitation phase [5,9]. However, no study reported a decline in the serum albumin level in only the convalescent rehabilitation phase; this decline did not seem to be an unusual matter. As fasting was involved in acute medical treatment in a certain period, a decline in serum albumin, perhaps, featured about a half-life of 20 days, which was not unusual.

Owing to the reasons for protein hyper metabolism, a study referred that the rehabilitation with discretion was necessary for stroke patients with poor nutrition condition [5]. In recovery phase rehabilitation wards, bedside rehabilitation and rehabilitation nursing were activities besides rehabilitation training rooms. Thus, further studies are warranted to investigate rehabilitation nursing as well as the therapy for poor nutrition condition.

In this study, the presence of dysphagia did not markedly correlate with the serum albumin level, perhaps, because of an effect of care and therapies by the multidisciplinary team approach. In the rehabilitation hospital, a regular meeting was held among involved carers, which possibly minimized any declining albumin level of patients with dysphagia. In addition, the presence of dysphagia markedly correlated 
Citation: Ikenishi K (2019) The Correlation between Dysphagia with Serum Albumin Level and Activities of Daily Living Improvement among Stroke Rehabilitation Patients: A Retrospective Study. J Nurs Care 8: 474. doi:10.4172/2167-1168.1000474

Page 4 of 4

with the FIM score (admission, 1 month later, variation), and the FIM score increment 1 month later was higher in the dysphagia group than the no dysphagia group.

Some studies have reported that more increment in the ADL score of stroke patients markedly correlated with mild severity of neurological symptoms and higher ADL score at admission [12-15]. Thus, as patients without dysphagia also exhibited mild severity, the degree of severity of stroke patients, perhaps, correlated with the FIM score in this study.

Regarding the location of the stroke, patients in the no dysphagia group were only left. It cannot be denied that the feature of subjects affected the result of the analysis. For example, the presence of aphasia and difficulty in using dominant right hand might affect the recovery of ADL. A study reported that patients with higher brain dysfunction barely recuperated ADL of stroke patients [16]. Hence, further comprehensive studies with a large sample size are warranted to collect data about higher brain dysfunction.

Our sample sizes was small and unbalanced between two groups, because this retrospective survey was conducted with medical record while we checked any omissions or errors on several record items which related to our research. Moreover, because we collected only data with obtaining patients/their family, our sample size was not large and unbalanced between two groups. Nevertheless, our results had significantly difference between two groups. We considered it a remarkable result. However, it was a necessary to a further research with larger sample size for more reliability analysis.

As univariate analysis was done, possible confounding factors were uncontrolled by multivariate analysis. These factors might include various eating assistance and swallowing exercises for dysphagia patients. However, we had no any measuring methods for getting objectively data on such cares. It was necessary to measure these data objectively and analyse with controlling confounding factors.

\section{Conclusions}

Regarding stroke patients in a recovery phase rehabilitation ward, comparison of patients with dysphagia and no dysphagia reveals the following: FIM scores are markedly higher; serum albumin levels are not markedly different groups; and dysphagia stroke patient's average serum albumin level declines after 1 month.

\section{References}

1. Wakabayashi H (2011) Rehabilitation and clinical nutrition. Jpn J Rehabil Med 48: 270-281.
2. Shiraishi A, Yoshimura Y, Wakabayashi H, Tsuji Y (2018) Prevalence of stroke-related sarcopenia and its association with poor oral status in postacute stroke patients: Implications for oral sarcopenia. Clin Nutr 37: 204-207.

3. Nii M, Maeda K, Wakabayashi H, Nishioka S, Tanaka A (2016) Nutritional improvement and energy intake are associated with functional recovery in patients after cerebrovascular disorders. J Stroke Cerebrovasc Dis 25: 57-62.

4. Kokura Y, Maeda K, Wakabayashi H, Nishioka S, Higashi S (2016) High nutritional-related risk on admission predicts less improvement of functional independence measure in geriatric stroke patients: A retrospective cohort study. J Stroke Cerebrovasc Dis 25: 1335-1341.

5. Yasuhiro O, Toru H, Hiroshi K, Komobuchi M, Yamada K, et al. (2015) Relationship between serum albumin level and long-term prognosis in patients with cerebral apoplexy. Jpn J Rehabil Med 52: 550-554.

6. Dávalos A, Ricart W, Gonzalez-Huix F, et al. (1996) Effect of malnutrition after acute stroke on clinical outcome. Stroke 27: 1028-1032.

7. Yoo SH, Kim JS, Kwon SU, Yun SC, Koh JY, et al. (2008) Under nutrition as a predictor of poor clinical outcomes in acute ischemic stroke patients. Arch Neurol. 65: 39-43.

8. Hopkins B (1993) Assessment of nutritional status. Nutrition support dietetics-core curriculum. (2ndedn), American Society for Parenteral and Enteral Nutrition.

9. Kim S, Byeon Y (2014) Comparison of nutritional status indicators according to feeding methods in patients with acute stroke. Nutr Neurosci 17: 138-144.

10. Aptaker RL, Roth EJ, Reichhardt G, Duerden ME, Levy CE (1994) Serum albumin level as a predictor of geriatric stroke rehabilitation outcome. Arch Phys Med Rehabil 75: 80-84.

11. Idicula TT, Waje-Andreassen U, Brogger J, Naess H, Thomassen L (2009) Serum albumin in ischemic stroke patients: the higher the better. The bergen stroke study. Cerebrovasc Dis 28: 13-17.

12. Yoneda Y, Uehara T, Yamasaki H, Kita Y, Tabuchi M, et al. (2003) Hospital-based study of the care and cost of acute ischemic stroke in Japan. Stroke 34: 718-724.

13. Saxena SK, Ng TP, Yong D, Fong NP, Gerald K (2006) Total direct cost, length of hospital stay, institutional discharges and their determinants from rehabilitation settings in stroke patients. Acta Neurol Scand 114: 307-314.

14. Cramer SC, Parrish TB, Levy RM, Stebbins GT, Ruland SD, et al. (2007) Predicting functional gains in a stroke trial. Stroke 38: 2108-2114.

15. Finestone HM, Greene-Finestone LS, Wilson ES, Teasell RW (1996) Prolonged length of stay and reduced functional improvement rate in malnourished stroke rehabilitation patients. Arch Phys Med Rehabil 77: 340-345.

16. Pettersen R, Dahl T, Wyller TB (2002) Prediction of long-term functional outcome after stroke rehabilitation. Clin Rehabil 16: 149-159. 